\title{
CONTROLLED LONGITUDINAL EMITTANCE BLOW-UP IN THE CERN PS
}

\author{
H. Damerau*, M. Morvillo, E. Shaposhnikova, J. Tuckmantel, J.-L. Vallet, \\ CERN, Geneva, Switzerland
}

\begin{abstract}
The longitudinal emittance of the bunches in the CERN PS must be increased before transition crossing to avoid beam loss due to a fast vertical instability. This controlled blow-up is essential for all high-intensity beams in the PS, including those for transfer to the LHC. The higher harmonic $200 \mathrm{MHz}$ RF system (six cavities) used for this blow-up has to generate a total RF voltage which, for the most demanding blow-up, is comparable to the voltage of the main RF cavities. The system is presently subject to a major upgrade and a possible reduction in the number of higher harmonic RF cavities installed is under consideration. To determine the minimum required, detailed simulations and machine development studies to optimize the longitudinal blow-up have been performed. Further options to produce the required longitudinal emittance using other RF systems are also analyzed. The results obtained for the different scenarios for the longitudinal blow-up are presented and compared in this paper.
\end{abstract}

\section{INTRODUCTION}

Thorough control of the longitudinal emittance, $\varepsilon_{l}$ to a high precision is required in the CERN PS to deliver stable beams with well-defined, constant parameters to the experiments and the subsequent accelerators. Of special importance are the various beams delivered to the LHC, which demand bunches of intensities from $5 \cdot 10^{9}$ to $1.15 \cdot 10^{11} \mathrm{ppb}$ with a longitudinal emittance, $\varepsilon_{l}$ between 0.2 (probe) and $0.35 \mathrm{eVs}$ (nominal). The bunches from the PS Booster are split in three parts at flat-bottom and in four at the flat-top, ideally dividing the longitudinal emittance by 12 . However, to keep the bunches stable in the PS and digestible for SPS, $\varepsilon_{l}$ must be increased with controlled longitudinal blow-ups (BU, see Fig. 1) [1].

\section{RF SYSTEM FOR BLOW-UP}

The longitudinal $\mathrm{BU}$ is performed by a higher harmonic (HH) $200 \mathrm{MHz}$ RF system, while the principal RF system is operated from 2.8 to $10 \mathrm{MHz}$. The RF system for BU presently consists of six cavities, each connected to a $25 \mathrm{~kW}$ power amplifier. As no high quality factor is required, the cavities are operated in air while only the acceleration gap is kept in vacuum. To allow for a sufficiently large bandwidth for modulation, the quality factor is reduced to $Q \simeq 2500$ by a strongly coupled external load.

\footnotetext{
*heiko.damerau@cern.ch
}

05 Beam Dynamics and Electromagnetic Fields

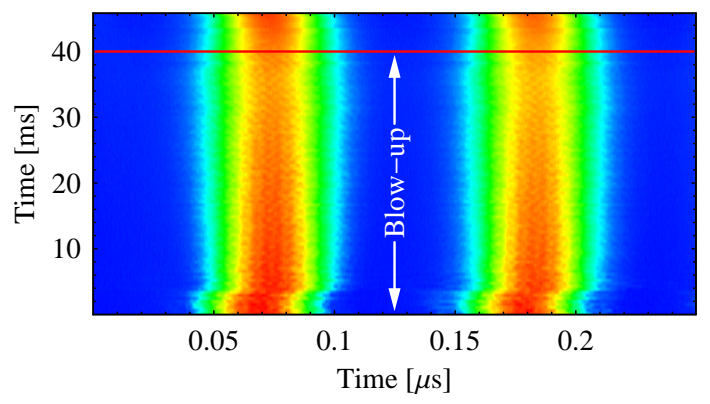

Figure 1: First two bunches of an LHC type beam during the $40 \mathrm{~ms}$ of BU. The peak density decreases while the bunches get longer. The bunch then shortens and peaks at constant emittance during acceleration.

When not operated, the cavities are short-circuited by three PIN diode switches coupled via $\lambda / 4$ lines.

Other RF systems in the PS could also be operated to blow up the beam longitudinally. To optimize the BU, studies have been performed in 2006. The results are presented for a BU with the $200 \mathrm{MHz} R F$ system, as well as for the alternative to use the $80 \mathrm{MHz}$ RF system for BU, normally operated for bunch rotation of LHC-type beams.

A simplified diagram of the PS $200 \mathrm{MHz}$ RF system during the low-energy BU $\left(E_{\text {kin }}=1.4 \mathrm{GeV}\right)$ is sketched in Fig. 2. In this case, the beam is steered so that the 458 th

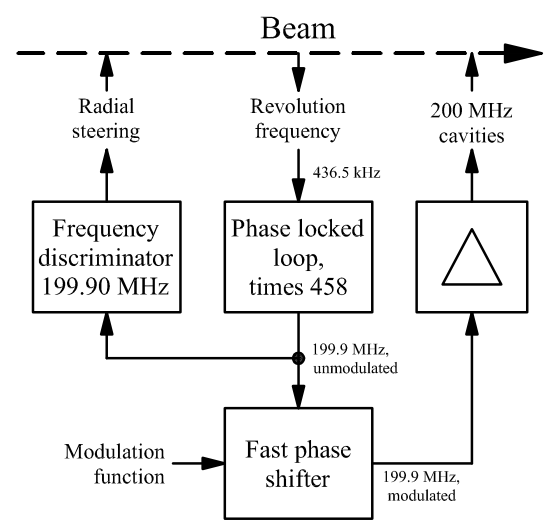

Figure 2: PS $200 \mathrm{MHz}$ system during BU at injection flatbottom. The radial loop is closed.

harmonic of the revolution frequency gets close to the resonance frequency of the $200 \mathrm{MHz}$ cavities.

Further BUs take place at an intermediate flat-top at $3.5 \mathrm{GeV} / \mathrm{c}$ and at flat-top energy of $14 / 26 \mathrm{GeV} / \mathrm{c}$. The numD02 Non-linear Dynamics - Resonances, Tracking, Higher Order 
ber of cavities needed for the different BUs varies from one to five, depending on the total voltage required for a certain beam type. However, the BU at low energy is the most demanding in terms of RF voltage.

The maximum RF voltage per $200 \mathrm{MHz}$ cavity (limited by the amplifier) depends on the phase modulation frequency, $f_{\bmod }$ and modulation depth, $\Delta \phi_{\bmod }$. For $f_{\bmod }=$ $10 \mathrm{kHz}$ and $\Delta \phi_{\bmod }=\pi$, the limitation was about $5 \mathrm{kV}$ in 2006. Recent improvements of the low-level signal generation show that more than 1.5 times that voltage per cavity can be reached now.

During the $\mathrm{BU}$, the longitudinal emittance grows by $\varepsilon_{f} / \varepsilon_{i} \simeq 1.5$ during 20 or $40 \mathrm{~ms}\left(\varepsilon_{i}, \varepsilon_{f}\right.$ : initial, final longitudinal emittance).

\section{BLOW-UP REGIMES}

In a double harmonic RF system, the total voltage acting on the beam can be written as

$$
V=V_{0} \sin \left(h_{0} \omega_{0} t\right)+V_{b} \sin \left(h_{b} \omega_{0} t+\psi(t)+\psi_{0}\right),
$$

with a sinusoidal phase modulation function

$$
\psi(t)=\Delta \phi_{\bmod } \sin \left(2 \pi f_{\bmod } t\right) .
$$

The analysis of the experimental data shows that two types of longitudinal BU can be distinguished, depending on the choice of the parameters for the BU. For a ratio between the harmonic number of the RF system used for $\mathrm{BU} h_{b}$ and that of the main RF system $h_{0}$ below 15 and $f_{\text {mod }}$ of few times the synchrotron frequency $f_{s}$, the $\mathrm{BU}$ is due to resonant excitation of the bunch $[2,3]$. For larger modulation frequency, which becomes more efficient for larger harmonic number ratio $h_{b} / h_{0}$, the effect of the HH RF system approaches a BU with noise [4].

Injecting band limited noise into the phase loop of the principal RF system was also tried. However, available time in the PS $(20-40 \mathrm{~ms})$ is too short, considering $1 / f_{s}=$ $1.2 \mathrm{~ms}$, for a smooth reproducible BU.

\section{Resonant Regime}

In this case, the BU rate depends on the relative phase $\psi_{0}$ between $\mathrm{HH}$ and principal RF system [2] and $k=$ $f_{\text {mod }} / f_{s}$. In this so-called resonant BU regime, for noninteger $h_{b} / h_{0}$ the BU rate may then vary from bunch to bunch, according to the position in the machine. The maximum BU rate is reached for $k<5$ [3]. For high $h_{\mathrm{b}} / h_{0}$ and $k$, multiple resonances are excited simultaneously, resulting in a smooth BU of all bunches, as observed in the PS. Originally, the BU was performed at $k=3$, which is less efficient for large $h_{b} / h_{0}$.

\section{Noise Regime}

For large $k>10$, as presently used [5], noise theory becomes applicable [4] and the efficiency of the BU is independent on the relative phase between principal and BU RF system.

05 Beam Dynamics and Electromagnetic Fields
Indeed, no dependence of the BU rate on the bunch position within the batch could be measured in the PS on beams prepared for the anti-proton decelerator $\left(\mathrm{AD}, h_{b} / h_{0}=\right.$ $458 / 8)$ and LHC $\left(h_{b} / h_{0}=458 / 21\right)$. The harmonic number ratio is non-integer in both cases and $k \simeq 14$. Moreover, a smooth bunch length and emittance growth along the BU is observed.

\section{SIMULATION AND MEASUREMENTS}

A numerical simulation with the longitudinal tracking program ESME [6] has been set-up for quantitative comparison with the measurements, starting from a mismatched bunch at injection in the PS. An ensemble of macro-particles having the same longitudinal distribution as the measured tomographic reconstruction (Fig. 3) of the longitudinal phase space has been generated as initial condition for the tracking. Due to the mismatch in length and

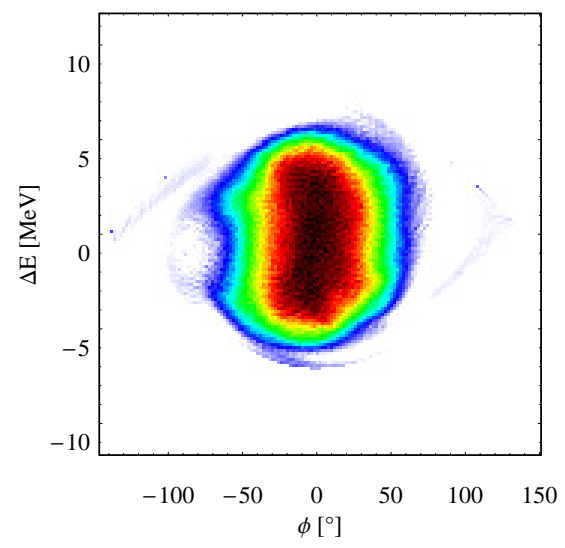

Figure 3: Tomographic reconstruction of the measured longitudinal distribution $1 \mathrm{~ms}$ after injection. The bunch is rotated by $90^{\circ}$ with respect to the time of injection. Longitudinal emittance: $\varepsilon_{\mathrm{RMS}}=0.26 \mathrm{eVs}$.

energy spread of the injected bunch, a strong quadrupole oscillation is excited. It decays during the first $10 \mathrm{~ms}$ after injection due to the non-linearity of the synchrotron motion. For comparison of the simulated BU with the measured data, the RMS bunch length is taken as a distribution independent observable.

\section{$200 \mathrm{MHz}$}

An example for the measured RMS bunch length during the $\mathrm{BU}$ at injection energy of $1.4 \mathrm{GeV}$ (kinetic) is shown in Fig. 4. The measurements have been performed with an $\mathrm{AD}$ type beam (four bunches injected into consecutive buckets at $h=8$ ). The intentional mismatch at injection is clearly visible in the form of quadrupole oscillations at $2 f_{s}$ ( $f_{s} \simeq 0.8 \mathrm{kHz}$ ). During the BU, the bunch length increases smoothly to $\tau_{\mathrm{RMS}}=45 \mathrm{~ns}$. The BU ends after $20 \mathrm{~ms}$ and the bunches get shorter again as acceleration starts. The parameters of the $200 \mathrm{MHz}$ system were $f_{\text {mod }}=10 \mathrm{kHz}$, $\Delta \phi_{\text {mod }}=\pi$ and $V_{b}=28 \mathrm{kV}$ (set-value).

D02 Non-linear Dynamics - Resonances, Tracking, Higher Order 


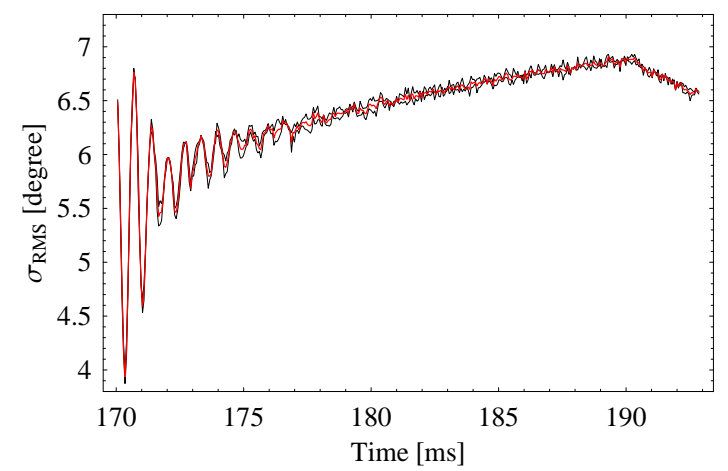

Figure 4: Measured RMS bunch length along the BU with $V_{b}=28 \mathrm{kV}$ at $200 \mathrm{MHz}, f_{\mathrm{mod}}=10 \mathrm{kHz}, \Delta \phi=3.1 \mathrm{rad}$; black lines: individual cycle, red line: average over several cycles.

Fig. 5 illustrates the simulated increase of $\tau_{\mathrm{RMS}}$, but one needs $V_{b}=37.5 \mathrm{kV}$ to match the measured bunch length behavior. A similar decay of the quadrupole oscillations is observed and the bunch length increases as smoothly as in the measurements. The results of the simulations suggest that the voltage in the cavities is some $35 \%$ higher than assumed, which is possible as no precise enough voltage calibration is presently available for the $200 \mathrm{MHz}$ cavities.

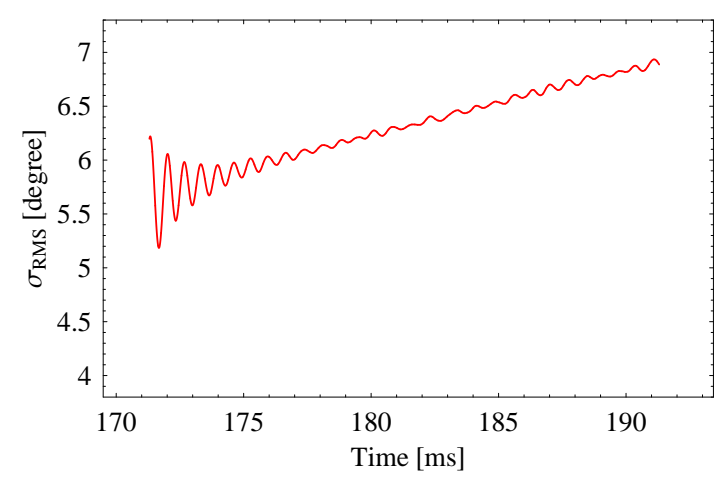

Figure 5: Simulated development of the RMS bunch length along the BU. Same BU parameters as for Fig. 4 , but $V_{b}=$ $37.5 \mathrm{kV}$.

\section{$80 \mathrm{MHz}$}

Similar tests have been done with the $80 \mathrm{MHz}$ RF system normally used for bunch shortening of LHC-type beams. Despite the high quality factor, $Q_{0}=22600$, their effective $Q$ is lowered by a fast feedback to about $Q_{\text {eff }} \simeq 100$ so that they can be operate with a phase-modulated RF signal at reduced voltage. The measured and simulated growth of $\tau_{\text {RMS }}$ is shown in Fig. 6. The parameters have been chosen such that the same BU as with the $200 \mathrm{MHz}$ system is achieved. The ESME simulation reproduces the measured bunch length increase without any arbitrary scaling factor for $V_{b}$. It is worth noting that the voltage of the $80 \mathrm{MHz}$ cavities is well calibrated.

05 Beam Dynamics and Electromagnetic Fields

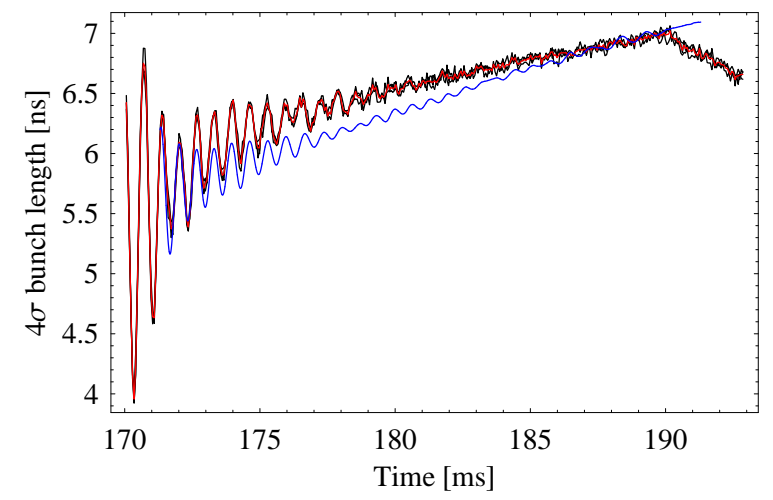

Figure 6: Comparison of measured RMS bunch length (black, average: red) during the $\mathrm{BU}\left(V_{b}=16 \mathrm{kV}\right.$ at $80 \mathrm{MHz}, f_{\text {mod }}=10 \mathrm{kHz}, \Delta \phi=2.45 \mathrm{rad}$ ) with the ESME simulation (blue) under the same conditions.

\section{CONCLUSION}

Controlled longitudinal emittance $\mathrm{BU}$ is required in the CERN PS to reliably handle high intensity beams close to their instability limitations. For the present parameters the effect of the phase modulated HH RF system can be approximated as noise. A simulation using a measured longitudinal distribution as initial condition has been performed with ESME. The comparison with beam measurements for BU with 80 and $200 \mathrm{MHz}$ RF system suggests that the voltage in the $200 \mathrm{MHz}$ cavities is $35 \%$ higher than previously assumed. BU using the $80 \mathrm{MHz} \mathrm{RF}$ system has been demonstrated. However, the required detuning of the cavity is incompatible with keeping it as spare for bunch rotation.

We would like to thank Milorad Popovic (Fermilab) for stimulating discussions and help during his stay at CERN.

\section{REFERENCES}

[1] D. Boussard, E. Brouzet, R. Cappi and J. Gareyte, "Collective Effects at Very High Intensity in the CERN-PS", IEEE Trans. Nucl. Sci, Vol. NS-26, 1979, p. 3568-3570.

[2] V. V. Balandin, M. B. Dyachkov, E. N. Shaposhnikova, "The Resonant Theory of Longitudinal Emittance BU by PhaseModulated High Harmonic Cavities", Part. Acc., Vol. 35, 1991, pp. 1-14.

[3] R. Cappi, R. Garoby, E. N. Shaposhnikova, "Experimental Study of Controlled Longitudinal BU”, CERN/PS 92-40 (RF), CERN, Geneva, Switzerland, 1992.

[4] D. Boussard, "The RF Noise Theory Applied to the PS Longitudinal Controlled Blow-up”, CERN SPS/ARF/Note 85-7, CERN, Geneva, Switzerland, 1985.

[5] S. Hancock, E. Métral, "Ghost Bunches and Blow-up Losses with High-Intensity Beams", CERN PS/RF Note 2002-198, CERN, Geneva, Switzerland.

[6] J. A. MacLachlan, J.-F. Ostiguy, "User's Guide to ESME 2003”, FNAL, Batavia, Illinois, 2003.

D02 Non-linear Dynamics - Resonances, Tracking, Higher Order 